\title{
Development of Weather Based Wheat Yield Forecast Models in Haryana
}

\author{
Sanjeev, Puneet Verma and Urmil Verma* \\ Department of Mathematics \& Statistics, CCS Haryana Agricultural University, \\ Hisar-125004, India \\ *Corresponding author
}

Keywords

Linear time trend, Eigen value, Eigen vector, Weather variables, Multicollinearity, Principal component score

Article Info

Accepted: 20 November 2018 Available Online: 10 December 2018
Parameter estimation in statistical modelling plays a crucial role in the real world phenomena. Several alternative analyses may be required for the purpose. An attempt has been made in this paper to estimate the yield of wheat crop using principal components of the weather parameters spread over the crop growth period. Principal component analysis has been used for the purpose of developing zonal yield forecast models because of multicollinearity present among weather variables. The results indicate the possibility of district-level wheat yield prediction, 4-5 weeks ahead of the harvest time in Haryana, India. Zonal weather models had the desired predictive accuracy and provided considerable improvement in the district-level wheat yield estimates. The estimated yield(s) from the selected models indicated good agreement with State Department of Agriculture (DOA) wheat yield(s) in most of the districts.

\section{Introduction}

Crop yield is affected by technological change and weather variability. It can be assumed that the technological factors will increase crop yield smoothly through time and therefore, year or some other parameter of time can be used to study the overall effect of technology on yield. Weather variables affect the crop differently during different stages of development. This increases the number of variables in the model and thus, a technique based on relatively smaller number of manageable parameters and at the same time, taking care of entire weather distribution may solve the problem. Principal Component Analysis (PCA) in this direction was carried out for pre-harvest wheat yield estimation on agro-climatic zone basis in Haryana.

Some similar studies concerning this work viz., weather models developed by Mehta et al., (2000), Agarwal et al., (2001) and Ramasubramanian et al., (2004) were successfully used for forecasting yields of various crops at district as well as agroclimatic zone level in different states of India. Hoogenboom (2000), Kandiannan et al., (2002), Bazgeer et al., (2007), Esfandiary et 
al., (2009), Lobell and Burke (2010), Basso et al., (2012) etc. have used a series of weather predictors for crop yield forecasting. Verma $e t$ al., (2011 and 2016) and Goyal and Verma (2015) have used agromet/spectral indices for the development of crop yield models of different crops in Haryana (India). Azfar et al., (2015) used principal component analysis for rapeseed and mustard yield forecast models for Faizabad district of U.P. (India).

\section{Crop status and data description}

Wheat is one of the most important cereal crops in India as it forms a major constituent of the staple diet of a large part of the population. India is the second largest producer among wheat growing countries of the World (Source: www.mapsofindia.com/indiaagriculture).

Haryana occupies the third position in wheat production among the various states in India (www.agricoop.nic.in/statistics). Haryana is self-sufficient in food grains production and also one of the top contributors of food grains to the central pool. Wheat occupies the foremost position followed by rice, not only regarding acreage and production but also in the versatility in adopting different soils and climatic conditions.

The Haryana state comprised of 22 districts is situated between $74^{\circ} 25^{\prime}$ to $77^{\circ} 38^{\prime} \mathrm{E}$ longitude and $27^{\circ} 40^{\prime}$ to $30^{\circ} 55^{\prime} \mathrm{N}$ latitude. The total geographical area of the state is 44212 sq. km. The DOA wheat yield data published by Bureau of Economics and Statistics, Haryana were compiled for the period 1980-1981 to 2012-2013 for Ambala, Kurukshetra, Rohtak, Karnal, Jind, Sonepat, Gurgaon, Faridabad, Mahendergarh, Hisar, Sirsa and Bhiwani districts, 1989-1990 to 2012-2013 for Yamunanagar, Panipat, Kaithal and Rewari, 1995-1996 to 2012-2013 for Panchkula, 1997-1998 to 2012-2013 for Jhajjar and Fatehabad districts and the same were used to carry out linear time-trend analysis and then computing the district-level trend based yield $\operatorname{Tr}=\mathrm{a}+\mathrm{bt}$, where $\operatorname{Tr}=$ Trend yield, $\mathrm{a}=$ Intercept, $\mathrm{b}=$ Slope and $\mathrm{t}=$ Year. The weather data for the last 33 years (1980-1981 to 2012-2013) were collected from India Meteorological Department (IMD) and different meteorological observatories of Haryana.

Weather data starting from $1^{\text {st }}$ fortnight of November to 1 month before harvest were utilized for the model building (crop growth period: $1^{\text {st }}$ November to $15^{\text {th }}$ April). Since the climatic data from adequate number of stations were not available; the districts having equable climatic conditions were grouped into four agro-climatic zones based on their physiography/soils and agro-climatic conditions in Haryana viz., zone-1: Ambala, Panchkula, Yamuna Nagar, Kurukshetra, zone-2: Karnal, Kaithal, Jind, Panipat, Sonipat, Rohtak, zone-3: Mahendergarh, Rewari, Jhajjar, Gurgoan, Faridabad and zone4: Sirsa, Fatehabad, Hisar, Bhiwani.

PC method was used for extraction of factors which consists of finding the eigen values and eigen vectors. Principal components (i.e. $\mathrm{P}_{\mathrm{i}}$, $\mathrm{i}=1,2, \ldots$ ) were obtained as $\mathrm{P}=\mathrm{kX}$, where $\mathrm{P}$ and $X$ are the column vectors of transformed and original variables and $\mathrm{k}$ is the matrix with rows as the characteristic vectors of the correlation matrix $R$. The variance of $P_{i}$ is the $\mathrm{i}^{\text {th }}$ characteristic root $\lambda_{\mathrm{i}}$ of the correlation matrix $\mathrm{R} ; \lambda_{\mathrm{s}}$ were obtained by solving the equation $|R-\lambda I|=0$. For each $\lambda$, the corresponding characteristic vector $\mathrm{k}$ was obtained by solving $|\mathrm{R}-\lambda \mathrm{I}| \mathrm{k}=0$

\section{Results and Discussion}

PC method consists of finding the eigen roots and eigen vectors of the correlation matrix of explanatory variables. 
Table.1 Eigen values and variance (\%) explained by different principal components

\begin{tabular}{|c|c|c|c|c|}
\hline \multirow[t]{2}{*}{ Components } & \multicolumn{4}{|c|}{ Eigen value (\% variance explained) } \\
\hline & Zone 1 & Zone 2 & Zone 3 & Zone 4 \\
\hline 1 & $4.91(18.17)$ & $5.10(18.90)$ & $4.48(16.59)$ & 4.52(16.75) \\
\hline 2 & $3.48(12.90)$ & $3.74(13.85)$ & $3.41(12.62)$ & 3.45(12.79) \\
\hline 3 & $2.67(09.89)$ & $3.02(11.19)$ & $2.73(10.12)$ & $2.77(10.25)$ \\
\hline 4 & $2.33(08.62)$ & $2.81(10.39)$ & $2.33(08.65)$ & $2.59(09.61)$ \\
\hline 5 & $2.24(08.28)$ & $2.07(07.67)$ & $2.11(07.81)$ & $2.09(07.74)$ \\
\hline 6 & $1.94(07.20)$ & $1.89(07.02)$ & $2.04(07.56)$ & $1.79(06.63)$ \\
\hline 7 & $1.73(06.40)$ & $1.48(05.48)$ & $1.71(06.34)$ & $1.62(05.99)$ \\
\hline 8 & $1.36(05.03)$ & $1.12(04.15)$ & $1.28(04.74)$ & 1.34(04.96) \\
\hline 9 & $1.24(04.61)$ & $1.06(03.92)$ & $1.17(04.34)$ & $1.10(04.08)$ \\
\hline 10 & $1.06(03.94)$ & $0.90(03.32)$ & $0.96(03.56)$ & $1.07(03.96)$ \\
\hline
\end{tabular}

Table.2 Zonal wheat yield models based on trend yield and PC scores

\begin{tabular}{|c|c|c|c|}
\hline $\begin{array}{l}\text { Model } \\
\text { variable }\end{array}$ & $\underline{\text { Zone1 }}$ & $\begin{array}{l}\text { Model } \\
\text { variable }\end{array}$ & $\frac{\text { Zone 2 }}{\text { Coefficients }}$ \\
\hline Constant & $\begin{array}{l}c \\
-2.93\end{array}$ & Constant & $\begin{array}{l}c \\
0.31\end{array}$ \\
\hline $\operatorname{Tr}$ & $\begin{array}{l}b_{1} \\
1.08\end{array}$ & $T r$ & $\begin{array}{c}b_{1} \\
0.99\end{array}$ \\
\hline$P C_{3}$ & $\begin{array}{l}b_{2} \\
0.77\end{array}$ & $P C_{1}$ & $\begin{array}{c}b_{2} \\
-0.73\end{array}$ \\
\hline$P C_{4}$ & $\begin{array}{l}b_{3} \\
-0.81\end{array}$ & $P C_{2}$ & $\begin{array}{l}b_{3} \\
0.81\end{array}$ \\
\hline$P C_{6}$ & $\begin{array}{l}b_{4} \\
0.85\end{array}$ & $\mathbf{P C}_{7}$ & $\begin{array}{l}b_{4} \\
-0.51\end{array}$ \\
\hline $\begin{array}{r}R^{2}=0.880 \\
\text { adj. } R^{2}=0.875\end{array}$ & $\mathrm{SE}=2.64$ & $\begin{array}{r}R^{2}=0.911 \\
\text { adj. } R^{2}=0.909\end{array}$ & $\mathrm{SE}=2.14$ \\
\hline
\end{tabular}




\begin{tabular}{|c|c|c|c|}
\hline $\begin{array}{c}\text { Model } \\
\text { variable }\end{array}$ & $\stackrel{\text { Zone } 3}{\text { Coefficients }}$ & $\begin{array}{l}\text { Model } \\
\text { variable }\end{array}$ & $\frac{\text { Zone 4 }}{\text { Coefficients }}$ \\
\hline Constant & $\begin{array}{c}C \\
1.31\end{array}$ & Constant & $\begin{array}{l}c \\
-1.78\end{array}$ \\
\hline$T r$ & $\begin{array}{c}b_{1} \\
0.99\end{array}$ & $T r$ & $\begin{array}{l}b_{1} \\
1.05\end{array}$ \\
\hline$P C_{3}$ & $\begin{array}{c}b_{2} \\
0.84\end{array}$ & $P C_{6}$ & $\begin{array}{c}b_{2} \\
-1.10\end{array}$ \\
\hline$P C_{6}$ & $\begin{array}{c}b_{3} \\
-0.50\end{array}$ & $P C_{10}$ & $\begin{array}{l}b_{3} \\
1.02\end{array}$ \\
\hline $\begin{array}{r}R^{2}=0.860 \\
\text { adj. } R^{2}=0.857\end{array}$ & $\mathrm{SE}=2.55$ & $\begin{array}{r}R^{2}=0.827 \\
\text { adj. } R^{2}=0.822\end{array}$ & $\mathrm{SE}=2.93$ \\
\hline
\end{tabular}

Zone 1: Yield est $_{1}=\left\{c+b_{1} \times T r+b_{2} \times P_{3}+b_{3} \times P_{4}+b_{4} \times P_{6}\right\}$

Zone 2: Yield est $=\left\{c+b_{1} \times T r+b_{2} \times P_{1}+b_{3} \times P_{2}+b_{4} \times P_{7}\right\}$

Zone 3: Yield est $=\left\{c+b_{1} \times \mathrm{Tr}+\mathrm{b}_{2} \times \mathrm{PC}_{3}+\mathrm{b}_{3} \times \mathrm{PC}_{6}\right\}$

Zone 4: Yield est $=\left\{c+b_{1} \times \mathrm{Tr}+\mathrm{b}_{2} \times \mathrm{PC}_{6}+\mathrm{b}_{3} \times \mathrm{PC}_{10}\right\}$

Where,

Yield $_{\text {est }}$ - Model predicted yield

c - Constant

$b_{i}$ - Regression coefficient $(i=1,2,3, \ldots$.

$\mathrm{T}_{\mathrm{r}}$ - Linear time trend based yield

$\mathrm{PC}_{\mathrm{i}}-\mathrm{i}^{\text {th }}$ principal component score $(\mathrm{i}=1,2, \ldots, 10)$

SE - Standard error of yield estimate

$\mathrm{R}^{2}$ - Coefficient of determination

Table.3 District-specific wheat yield estimates alongwith percent deviations from DOA yield(s) using fitted models

\begin{tabular}{|c|c|c|c|c|c|c|c|c|c|}
\hline \multirow{2}{*}{$\begin{array}{l}\text { Districts/ } \\
\text { Years }\end{array}$} & \multicolumn{3}{|c|}{ Ambala } & \multicolumn{3}{|c|}{ Kurukshetra } & \multicolumn{3}{|c|}{ Yamunanagar } \\
\hline & $\begin{array}{l}\text { Obs. } \\
\text { Yield } \\
\text { (q/ha) }\end{array}$ & $\begin{array}{l}\text { Fitted } \\
\text { Yield } \\
\text { (q/ha) }\end{array}$ & (RD\%) & $\begin{array}{l}\text { Obs. } \\
\text { Yield } \\
\text { (q/ha) }\end{array}$ & $\begin{array}{l}\text { Fitted } \\
\text { Yield } \\
\text { (q/ha) }\end{array}$ & (RD\%) & $\begin{array}{l}\text { Obs. } \\
\text { Yield } \\
\text { (q/ha) }\end{array}$ & $\begin{array}{l}\text { Fitted } \\
\text { Yield } \\
\text { (q/ha) }\end{array}$ & (RD\%) \\
\hline 2011 & .01 & & 10.34 & 8 & 93 & 4.51 & 53.64 & +3.56 & 18.80 \\
\hline 2012-13 & 42.06 & 42.30 & -0.58 & 46.57 & 50.66 & -8.77 & 43.39 & 42.15 & 2.85 \\
\hline
\end{tabular}

\begin{tabular}{|c|c|c|c|c|c|c|c|c|c|}
\hline \multirow{2}{*}{$\begin{array}{l}\text { Districts/ } \\
\text { Years }\end{array}$} & \multicolumn{3}{|c|}{ Rohtak } & \multicolumn{3}{|c|}{ Karnal } & \multicolumn{3}{|c|}{ Jind } \\
\hline & $\begin{array}{l}\text { Obs. } \\
\text { Yield } \\
\text { (q/ha) }\end{array}$ & $\begin{array}{l}\text { Fitted } \\
\text { Yield } \\
\text { (q/ha) }\end{array}$ & (RD\%) & $\begin{array}{l}\text { Obs. } \\
\text { Yield } \\
\text { (q/ha) }\end{array}$ & $\begin{array}{l}\text { Fitted } \\
\text { Yield } \\
\text { (q/ha) }\end{array}$ & (RD\%) & $\begin{array}{l}\text { Obs. } \\
\text { Yield } \\
\text { (q/ha) }\end{array}$ & $\begin{array}{l}\text { Fitted } \\
\text { Yield } \\
\text { (q/ha) }\end{array}$ & (RD\%) \\
\hline & 50.20 & 43. & & 56.7 & 49.24 & & 52.35 & 48.23 & 7. \\
\hline 2012-13 & 37.38 & 44.38 & -18.72 & 46.7 & 50.62 & -8.39 & 42.70 & 49.69 & -16.36 \\
\hline
\end{tabular}




\begin{tabular}{|c|c|c|c|c|c|c|c|c|c|}
\hline \multirow{2}{*}{$\begin{array}{l}\text { Districts/ } \\
\text { Years }\end{array}$} & \multicolumn{3}{|c|}{ Sonipat } & \multicolumn{3}{|c|}{ Panipat } & \multicolumn{3}{|c|}{ Kaithal } \\
\hline & $\begin{array}{l}\text { Obs. } \\
\text { Yield } \\
\text { (q/ha) }\end{array}$ & $\begin{array}{l}\text { Fitted } \\
\text { Yield } \\
\text { (q/ha) }\end{array}$ & (RD\%) & $\begin{array}{l}\text { Obs. } \\
\text { Yield } \\
\text { (q/ha) }\end{array}$ & $\begin{array}{l}\text { Fitted } \\
\text { Yield } \\
\text { (q/ha) }\end{array}$ & (RD & $\begin{array}{l}\text { Obs. } \\
\text { Yield } \\
\text { (q/ha) }\end{array}$ & $\begin{array}{l}\text { Fitted } \\
\text { Yield } \\
\text { (q/ha) }\end{array}$ & (RI \\
\hline & & & & 3017 & & & 54.51 & & 3.66 \\
\hline 2012-1 & 45.20 & 49.40 & -9.29 & 42.47 & 45.24 & -6.53 & 46.84 & 48.26 & -3.04 \\
\hline
\end{tabular}

\begin{tabular}{|c|c|c|c|c|c|c|c|c|c|}
\hline \multirow{2}{*}{$\begin{array}{l}\text { Districts/ } \\
\text { Years }\end{array}$} & \multicolumn{3}{|c|}{ Gurgaon } & \multicolumn{3}{|c|}{ Faridabad } & \multicolumn{3}{|c|}{ Mahendergarh } \\
\hline & $\begin{array}{l}\text { Obs. } \\
\text { Yield } \\
\text { (q/ha) }\end{array}$ & $\begin{array}{l}\text { Fitted } \\
\text { Yield } \\
\text { (q/ha) }\end{array}$ & (RD\%) & $\begin{array}{l}\text { Obs. } \\
\text { Yield } \\
\text { (q/ha) }\end{array}$ & $\begin{array}{l}\text { Fitted } \\
\text { Yield } \\
\text { (q/ha) }\end{array}$ & (RD\%) & $\begin{array}{l}\text { Obs. } \\
\text { Yield } \\
\text { (q/ha) }\end{array}$ & $\begin{array}{l}\text { Fitted } \\
\text { Yield } \\
(\mathbf{q} / \mathbf{h a})\end{array}$ & (RD\%) \\
\hline & & & & 37 & & & & & 0.93 \\
\hline 2012-13 & 5.54 & 44.07 & 3.2 & 45.39 & 45.82 & -0.94 & 47.77 & 45.74 & 4.25 \\
\hline
\end{tabular}

\begin{tabular}{|c|c|c|c|c|c|c|c|c|c|}
\hline \multirow{2}{*}{$\begin{array}{l}\text { Districts/ } \\
\text { Years }\end{array}$} & \multicolumn{3}{|c|}{ Rewari } & \multicolumn{3}{|c|}{ Jhajjar } & \multicolumn{3}{|c|}{ Hisar } \\
\hline & $\begin{array}{l}\text { Obs. } \\
\text { Yield } \\
\text { (q/ha) }\end{array}$ & $\begin{array}{l}\text { Fitted } \\
\text { Yield } \\
\text { (q/ha) }\end{array}$ & (RD\%) & $\begin{array}{l}\text { Obs. } \\
\text { Yield } \\
\text { (q/ha) }\end{array}$ & $\begin{array}{l}\text { Fitted } \\
\text { Yield } \\
\text { (q/ha) }\end{array}$ & (RD\%) & $\begin{array}{l}\text { Obs. } \\
\text { Yield } \\
\text { (q/ha) }\end{array}$ & $\begin{array}{l}\text { Fitted } \\
\text { Yield } \\
\text { (q/ha) }\end{array}$ & $(\mathrm{RD} \%)$ \\
\hline & & & & & & & 5008 & 49 & \\
\hline 2012-13 & 49.28 & 44.19 & 10.33 & 39.71 & 41.14 & -3.59 & 42.73 & 48.48 & -13.45 \\
\hline
\end{tabular}

\begin{tabular}{|c|c|c|c|c|c|c|c|c|c|}
\hline \multirow{2}{*}{$\begin{array}{l}\text { Districts/ } \\
\text { Years }\end{array}$} & \multicolumn{3}{|c|}{ Sirsa } & \multicolumn{3}{|c|}{ Bhiwani } & \multicolumn{3}{|c|}{ Fatehabad } \\
\hline & $\begin{array}{l}\text { Obs. } \\
\text { Yield } \\
\text { (q/ha) }\end{array}$ & $\begin{array}{l}\text { Fitted } \\
\text { Yield } \\
\text { (q/ha) }\end{array}$ & (RD\%) & $\begin{array}{l}\text { Obs. } \\
\text { Yield } \\
\text { (q/ha) }\end{array}$ & $\begin{array}{l}\text { Fitted } \\
\text { Yield } \\
\text { (q/ha) }\end{array}$ & (RD\%) & $\begin{array}{l}\text { Obs. } \\
\text { Yield } \\
\text { (q/ha) }\end{array}$ & $\begin{array}{l}\text { Fitted } \\
\text { Yield } \\
\text { (q/ha) }\end{array}$ & $(\mathrm{RD} \%)$ \\
\hline & 57 & & & & & & 54.72 & & 7.64 \\
\hline 2012-13 & 48.42 & 48.10 & 0.67 & 40.55 & 42.20 & -4.06 & 46.81 & 49.02 & -4.72 \\
\hline
\end{tabular}

Percent Relative Deviation $(\mathrm{RD} \%)=100 \times[$ (observed (obs.) yield - fitted yield) $/$ observed yield $]$

The most frequently used convention is to retain the components whose eigen values are greater than one. Weather data starting from first fortnight of November to first fortnight of March i.e. one month before crop harvest were utilized for the model development. In this study, first ten eigen values of the correlation matrix of explanatory variables (weather parameters) suggested ten factor(s) solution (Table 1). However, the remaining components accounted for a smaller amount of total variation. Hence, those components were not considered to be of much practical significance. Eigen vectors being the weights were used to compute PC scores. Multiple linear regression models via step-wise regression method (Draper and Smith, 2003) were fitted by considering PC scores as regressors and wheat yield as dependent variable. The models finalized as shown in Table 2 were used to obtain the district-level wheat yield estimates (Table 3).

Trend yield is an important parameter appearing in all the models, indicating that most of the variability in yield is explained by $T r$, which is an indication or technological advancement, improvement in fertilizer/ insecticide/pesticide/weedicide used and increased use of high yielding varieties. The predictive performance(s) of the fitted models were observed in terms of the percent deviations of wheat yield forecasts in relation to real time wheat yield(s). The estimated 
yield(s) from the selected models indicated good agreement with DOA wheat yield estimates in most of the districts.

\section{References}

Agarwal, R., Jain, R.C. and Mehta, S.C. (2001). Yield forecast based on weather variables and agricultural inputs on agroclimatic zone basis. Indian J. of Agril. Sci. 71(7), 487490.

Azfar, M., Sisodia, B. V. S., Rai, V. N. and Devi, M. (2015). Pre-harvest forecast models for rapeseed and mustard yield using principal component analysis of weather variables, Mausam, 66(4), 761-766.

Basso, B., C. Fiorentino, D. Cammarano, G. Cafiero and J. Dardanelli (2012). Analysis of rainfall distribution on spatial and temporal patterns of wheat yield in Mediterranean environment. European Journal of Agronomy, 41, 52- 65.

Bazgeer, S., Gh. Kamali and A. Mortazavi (2007). Wheat yield prediction through agrometeorological indices for Hamedan, Iran.Biaban, 12, 33-38.

Draper, N.R. and Smith, H. 2003. Applied Regression Analysis. $3^{\text {rd }}$ edition, John Wiley and Sons. New York.

Esfandiary, F., G. Aghaie and A. D. Mehr (2009). Wheat yield prediction through agro meteorological indices for Ardebil district. World Academy of Science: Engineering and Technology, 49, 32-35.

Goyal, M. and Verma, U. (2015). Development of weather-spectral models for pre-harvest wheat yield prediction on agro-climatic zone basis in Haryana, International J. of
Agricultural and Statistical Sciences, 11(1), 73-79.

Hoogenboom, G. (2000). Contribution of agrometeorology to the simulation of crop production and its applications. Agricultural and Forest Meteorology, 103, 137-157.

Kandiannan, K., K. K. Chandaragiri, N. Sankaran, T. N. Balasubramanian and C. Kailasam (2002). Crop-weather model for turmeric yield forecasting for Coimbatore District, Tamil Nadu, India. Agricultural and Forest Meteorology, 112, 133-137.

Lobell, D. B and M. Burke (2010). On the use of statistical models to predict crop yield responses to climate change. Agricultural and Forest Meteorology, 150, 1443-1452.

Mehta, S.C., Agarwal, R. and Singh, V.P.N. (2000). Strategies for composite forecast. $J$. Ind. Soc. Agril. Statist. 53(3), 262-272.

Ramasubramanian, V., Agrawal, R. and Bhar, L.M. (2004). Forecasting sugarcane yield using multiple Markov chains. (IASRI, New Delhi publication).

Verma, U., Dabas, D. S., Hooda, R. S., Kalubarme, M. H., Yadav, M. and Sharma, M. P. (2011). Remote Sensing Based wheat acerage and spectraltrend-agrometeorological yield forecasting: Factor Analysis Approach. Society of Statistics, Computers and Applications, 9(1and2), 1-13.

Verma, U., Piepho, H. P., Goyal, A., Ogutu, J. O. and kalubarme, M. H. (2016). Role of climatic variables and crop condition term for mustard yield prediction in Haryana (India). International J. of Agricultural and Statistical Sciences, 12(1), 45-51.

\section{How to cite this article:}

Sanjeev, Puneet Verma and Urmil Verma. 2018. Development of Weather Based Wheat Yield Forecast Models in Haryana. Int.J.Curr.Microbiol.App.Sci. 7(12): 2973-2978. doi: https://doi.org/10.20546/ijcmas.2018.712.340 